\title{
Stochastic Measure of Fatigue Crack Damage for Health Monitoring of Ductile Alloy Structures
}

\begin{abstract}
Asok Ray*
Mechanical Engineering Department, The Pennsylvania State University, University Park, PA 16802, USA

This paper models a stochastic measure of fatigue crack damage in ductile alloys that are commonly encountered in structures and machinery components of complex mechanical systems such as land, air, ocean, and space vehicles. The constitutive equations of the damage measure are built upon the physics of fracture mechanics and are substantiated by Karhunen-Loève decomposition of fatigue test data where statistical orthogonality of the estimated measure and the resulting estimation error is demonstrated in a Hilbert space setting. The non-stationary probability distribution (PDF) function of the damage estimate is generated in a closed form without numerically solving stochastic differential equations in the Wiener integral or Itô integral setting. The model of crack damage measure allows real-time execution of decision algorithms for health monitoring, risk assessment, and life prediction of mechanical structures on inexpensive platforms such as a Pentium processor. The stochastic model of fatigue crack damage measure is in good agreement with experimental data sets for 2024-T3 and 7075-T6 aluminum alloys.
\end{abstract}

Keywords stochastic modeling - fatigue crack growth · damage measure - risk analysis . life prediction

\section{Introduction}

Decision systems for health monitoring of mechanical structures are synthesized by taking mission objectives (e.g., productivity and performance), service life, and overall cost into consideration [23]. The current state-of-the-art of synthesizing decision systems for health monitoring and life prediction of operating machinery focuses on enhancement of reliability and diagnostic capabilities under constraints that often do not adequately represent the material

*Author to whom correspondence should be addressed.

E-mail: axr2@psu.edu degradation aspects of critical plant components [11]. The reason is that traditional design methodologies are usually based upon the assumption of invariant characteristics of structural materials. However, in reality, since structural integrity of critical components monotonically degrades with age and cycles of operation, the maintenance strategies for new and old machinery are likely to be significantly different even if they are identically operated. Therefore, it is highly desirable to update operation and maintenance strategies in real time as new information on

Copyright (C) 2004 Sage Publications,

Vol 3(3): 0245-263

[1475-9217 (200409) 3:3;245-263 10.1177/1475921704045626] 
sensor data and history of machinery operation and anticipated usage becomes available.

A concept of Information-Based Health and Usage Monitoring is that of updating decisions for inspection, repair, maintenance scheduling based on the evolving knowledge of operation history and anticipated loading of the machinery as well as the physical characteristics and dynamics of material degradation in critical components. The key steps in the synthesis of health monitoring strategies are formulations of:

- Physics-based dynamic models of material degradation including identification of failure precursors;

- Statistical models of hypothesis tests for risk analysis and remaining life prediction under different operating conditions;

- Decision algorithms for maintenance scheduling based on the information derived from operation history (e.g., sensor data and expert knowledge) and anticipated usage of the machinery.

From the above perspectives, stochastic modeling and analysis of fatigue crack phenomena in ductile alloys has received considerable attention over the last several decades. A list of the literature representing the state-of-the-art is cited by Sobczyk and Spencer [31] as well as in the March 1996 issue of Engineering Fracture Mechanics.

Bogdonoff and Kozin [5] proposed a Poissonlike independent-increment jump model of fatigue crack phenomena. The underlying principle of this model agrees with the theory of micro-level fatigue cracking. An alternative approach to stochastic modeling of fatigue crack damage is to randomize the coefficients of an existing deterministic model to represent material inhomogeneity [7]. Another alternative approach is to augment a deterministic model of fatigue crack growth with a random process $[12,16,32]$. The fatigue crack growth process is thus modeled by nonlinear stochastic differential equations in the Itô setting $[15,22]$. Specifically, Kolmogorov forward and backward diffusion equations, which require solutions of nonlinear partial differential equations, have been proposed to generate the statistical information required for risk analysis of mechanical structures [4,34]. These nonlinear partial differential equations can only be solved numerically and the numerical procedures are computationally intensive as they rely on fine-mesh models using finite-element or combined finitedifference and finite-element methods [31]. Casciati et al. [6] have analytically approximated the solution of Itô equations by Hermite moments to generate a probability distribution function of the crack length.

This paper presents a stochastic measure of fatigue crack damage in ductile alloys that are commonly encountered in structures and machinery components of complex mechanical systems (e.g., land, air, ocean, and space vehicles). The measure of fatigue crack damage at an instant (i.e., at the end of a stress cycle) is expressed as a continuous function of the current and initial crack lengths in the mean square $(m s)$ sense. The non-stationary probability distribution of an estimated measure of fatigue crack damage is obtained in a closed form without numerically solving stochastic differential equations in the Wiener integral or Itô integral setting. Model predictions are shown to be in close agreement with the fatigue test data of 2024-T3 and 7075-T6 aluminum alloys. The paper also illustrates how the stochastic damage measure can be used for condition monitoring as well as in making decisions for risk analysis and life prediction that are necessary for health management and life extending control of mechanical and aerospace systems [25]. The proposed model of stochastic damage measure would require parameter identification and tuning in a laboratory environment, prior to its implementation for health monitoring in a mechanical structure that may have its own unique material property and crack geometry. For example, Keller and Ray [14] have reported such experiments in a laboratory environment using optical microscopic and ultrasonic sensing devices for crack damage assessment.

\section{Measure of Fatigue Crack Damage}

Traditionally fatigue crack growth models have been formulated by fitting estimated mean values 
of fatigue crack length, generated from ensemble averages of experimental data, as functions of time in units of cycles [24,30]. Ray and Patankar [27] have formulated the state-space modeling concept of crack growth based on fracturemechanistic principles of the crack-closure concept. The state-space model has been validated by fatigue test data for variable-amplitude cyclic loading [30]. The following definition of a fatigue cycle is adopted for model development in the sequel.

Definition 2-1: The $k$ th fatigue cycle is defined on the time interval: $\Im_{k}=\left\{\tau \in \Re: \underline{\tau}_{k-1}<\tau \leq \underline{\tau}_{k}\right\}$ with $\underline{\tau}_{k-1}<\bar{\tau}_{k}<\underline{\tau}_{k}$ where $\underline{\tau}_{k}$ and $\bar{\tau}_{k}$ are the instants of occurrence of the minimum stress $S_{k}^{\min }$ and the maximum stress $S_{k}^{\max }$, respectively. The $k$ th fatigue cycle is denoted as the ordered pair $\left(S_{k}^{\max }, S_{k}^{\min }\right)$.

It follows from the above definition that $S_{k}^{\max }>\max \left(S_{k-1}^{\min }, S_{k}^{\min }\right)$.

A stress cycle is determined by the maximum stress $S^{\max }$ and the following minimum stress $S^{\mathrm{min}}$. The frequency and the shape of a stress cycle are not relevant for crack growth in ductile alloys at room temperature [1]. The load dependence of crack growth is assumed to be completely characterized by peaks and valleys of applied stress at temperatures significantly below about one-third of the melting point (e.g. room temperature for aluminum and ferrous alloys).

Following Sobczyk and Spencer [31] and the pertinent references cited therein, the stochastic model of fatigue crack damage, formulated in this paper, is built upon the state-space structure of the mean-value model [27] that accounts for variable-amplitude cyclic stress and crack retardation phenomena [21]:

$$
\begin{aligned}
& \delta \hat{a}_{t} \equiv \hat{a}_{t}-\hat{a}_{t-\delta t}=h\left(\Delta K_{t}^{e f f}\right) \delta t \text { with } h(0)=0 ; \\
& \text { for } t \geq t_{\mathrm{o}} \text { and given } \hat{a}_{t_{\mathrm{o}}}>0 \\
& \Delta K_{t}^{\mathrm{eff}}=\Delta S_{t}^{e} \sqrt{\pi \hat{a}_{t-\delta t}} F\left(\hat{a}_{t}\right) \\
& \Delta S_{t}^{e}=\left[S_{t}^{\max }-\max \left(S_{t}^{\mathrm{o}}, S_{t-\delta t}^{\min }\right)\right] U\left(S_{t}^{\max }-S_{t-\delta t}^{\mathrm{o}}\right)
\end{aligned}
$$

where $t$ is the current time upon completion of a stress cycle, and $t_{0}$ is the initial time (e.g., when the machine component is put in service after a major maintenance or inspection); $\hat{a}(t)$ is the estimated mean value of (time-dependent) crack length; $\delta \hat{a}(t)$ is the increment of the estimated mean crack length over one cycle at time $t$, and $\delta t$ indicates the time increment over the cycle completed at time $t ; h(\cdot)$ is a non-negative Lebesgue-measurable function [2] which is dependent on the material and geometry of the stressed component; and $\Delta S_{t}^{e}$ is the effective stress range during the cycle completed at time $t$ with the corresponding crack opening stress $S_{t}^{\mathrm{o}}$, maximum stress $S_{t}^{\max }$, and minimum stress $S_{t}^{\min }$; and the Heaviside unit step function $U(\cdot)$ is defined as:

$$
U(x)= \begin{cases}0 & \text { if } x<0 \\ 1 & \text { if } x \geq 0\end{cases}
$$

The (dimensionless) correction factor $F$ is dependent on geometrical configuration (e.g., thickness, width, and the crack type in the stressed component) and the crack length. For example, $F=\sqrt{\sec (\pi \hat{a}(t) /(2 w))}$ for center-cracked specimens of half-width $w$ for which $0<\hat{a}(t)<w$ at every $t \geq t_{0}$.

There are several empirical and semi-empirical methods for calculating the crack opening stress $S^{0}$. For example, Newman [20] has formulated an algebraic relation to obtain $S^{0}$ as a function of peak stress $S^{\max }$ and stress ratio $\mathrm{R} \equiv S^{\mathrm{min}} / S^{\max }$ under constant-amplitude cyclic loading. The estimated mean $\hat{a}$ of crack length is normalized with respect to the parameter of its physical dimension (e.g., half-width for centercracked specimens or full width for compact specimens) to obtain the estimated mean of a dimensionless crack length defined as:

$$
\hat{c}_{t} \equiv \frac{\hat{a}_{t}}{w} \Rightarrow 0<\hat{c}_{t}<1 \quad \forall t \geq t_{\mathrm{o}}
$$

and the effective stress range $\Delta S_{t}^{e}$ is normalized with respect to the flow stress $S^{\text {flow }}$ (which is the average of yield stress and the ultimate strength of the structural material) as: 


$$
\Delta S_{t} \equiv \frac{\Delta S_{t}^{e}}{S^{\text {flow }}}
$$

It has been shown in the fracture mechanics literature [1] that, for a given geometry (i.e., thickness and width) of center-cracked specimens of ductile alloys, the function $h(\cdot)$ in Equation (1) is separable as a product of two functions, $h_{1}\left(\Delta S_{t}\right)$ and $h_{2}\left(\hat{c}_{t}\right)$. For $\hat{c}_{t} \ll 1$ in center-cracked specimens, Equation (1) can be approximated by series approximation of the cosine term in the correction factor $F$ as:

$$
\begin{aligned}
\delta \hat{c}_{t} & =\hat{\Omega}\left(\Delta S_{t} \sqrt{\frac{\hat{c}_{t}}{\cos \left(\pi / 2 \hat{c}_{t}\right)}}\right)^{m} \delta t \\
& \cong \hat{\Omega} \frac{\left(\Delta S_{t} \sqrt{\hat{c}_{t}}\right)^{m}}{1-m\left(\pi / 4 \hat{c}_{t}\right)^{2}} \delta t
\end{aligned}
$$

for $t \geq t_{0}$ and $0<\hat{c}_{t_{0}} \leq \hat{c}_{t}<4 /(\pi \sqrt{m})$ that ensures non-negativity of the crack length increment $\delta \hat{c}_{t}$. The constant dimensionless parameters $\hat{\Omega}$ and $m$ are dependent on the specimen material, geometry, and fabrication. For constant-amplitude loading, Equation (4) reduces to the wellknown Paris equation [24]. For varying-amplitude load, Ray and Patankar [27] have validated Equation (4) under varying load amplitude (i.e., time-dependent stress range $\Delta S_{t}$ by having $S^{0}(t)$ as a state variable.

Ditlevsen [7] has shown that, under constant load amplitude, the randomness of fatigue crack growth accrues primarily from parametric uncertainties. The stochastic process of crack growth is largely dependent on two second-order random parameters - a multiplicative process $\Omega(\zeta, \Delta S)$ and an exponent parameter $m(\zeta)$. Ditlevsen [7] suggested the possibility of one of the above two random variables being a constant for all specimen $\zeta$. Statistical analysis of the experimental data for 2024-T3 and 7075-T6 aluminum alloys by Ray and Tangarilla (1997) and Ray and Phoha (1999) reveals that the random exponent $m(\zeta)$ can be approximated as a constant for all specimens, i.e., having $m(\zeta)=m$ with probability (wp) 1, at different levels of constant stress range $\Delta S$ for a given material. Based on this observation and the (deterministic) model structure in Equation (4), we postulate the following constitutive equation for fatigue crack growth in the stochastic setting [31] partly similar to what was originally proposed by [24] in the deterministic setting:

$$
\begin{aligned}
& \delta c_{t}(\zeta)=\Omega\left(\zeta, \Delta S_{t}\right)\left(\frac{\left(\Delta S_{t} \sqrt{c_{t}(\zeta)}\right)^{m}}{1-m\left(\pi / 4 c_{t}(\zeta)\right)^{2}}\right) \rho_{t}(\zeta) \delta t \\
& \quad \text { for } t \geq t_{\mathrm{o}} \text { and } 0<c_{t_{\mathrm{o}}}(\zeta) \leq c_{t}(\zeta)<\frac{4}{\pi \sqrt{m}} w p 1
\end{aligned}
$$

where the (at least) second-order random process $\Omega\left(\zeta, \Delta S_{t}\right)$ is pertinent to a test specimen $\zeta$ for a (possibly varying amplitude) stress range $\Delta S_{t}$, and represents uncertainties in manufacturing (e.g., machining); $\Omega\left(\zeta, \Delta S_{t}\right)$ is assumed to be independent of material microstructure; and the (at least) second-order (positively correlated) stochastic process $\rho_{t}(\zeta)$ represents (multiplicative) uncertainties in the material microstructure and crack length measurements that may vary with crack propagation in a given specimen $\zeta$. We postulate that the process $\rho_{t}\left(\zeta, \Delta S_{t}\right)$ is statistically independent of $\Omega\left(\zeta, \Delta S_{t}\right)$ for all $t \geq t_{0}$. The rationale for this independence assumption is that inhomogeneity of the material microstructure and measurement noise associated with each test specimen, represented by $\rho_{t}(\zeta)$, are unaffected by the uncertainty $\Omega\left(\zeta, \Delta S_{t}\right)$ due to machining operations, for example. Without loss of generality, we constrain that $E\left[\rho_{t}(\zeta)\right]=1 \forall t \geq t_{\mathrm{o}}$. Furthermore, non-negativity of crack length increment $\delta c_{t}(\zeta)$ is assured in the almost sure (as) sense by imposing the constraints $\Omega\left(\zeta, \Delta S_{t}\right) \geq 0$ and $\rho_{t}(\zeta) \geq 0 \mathrm{wp} \quad \forall t \geq t_{\mathrm{o}}$.

Since the number of cycles to failure is usually very large in the crack growth processes (even for low-cycle fatigue), a common practice in the fracture mechanics literature is to approximate the difference equation of crack growth by a differential equation. Therefore, for $t \geq t_{0}$ and $c_{t_{0}}(\zeta)>0 \mathrm{wp} 1$, the crack growth increment in Equation (5) is approximated as the Riemann sum that is obtained as a stochastic differential equation in the almost sure (as) sense: 


$$
\begin{array}{r}
\left(c_{t}(\zeta)^{-m / 2}-m\left(\frac{\pi}{4}\right)^{2} c_{t}(\zeta)^{2-m / 2}\right) d c_{t}(\zeta) \\
=\left(\Delta S_{t}\right)^{m} \Omega\left(\zeta, \Delta S_{t}\right) \rho_{t}(\zeta) d t \quad w p 1
\end{array}
$$

which is integrated pointwise (i.e., for the individual $\zeta$ 's) as follows:

$$
\begin{aligned}
& \int_{c_{t_{0}}(\zeta)}^{c_{t}(\zeta)} \frac{d \xi}{\xi^{m / 2}}-m\left(\frac{\pi}{4}\right)^{2} \int_{c_{t_{0}}(\zeta)}^{c_{t}(\zeta)} \frac{d \xi}{\xi^{-2+m / 2}} \\
& =\int_{t_{\mathrm{o}}}^{t}\left(\Delta S_{\tau}\right)^{m} \Omega\left(\zeta, \Delta S_{\tau}\right) \rho_{\tau}(\zeta) d \tau \text { wp } 1
\end{aligned}
$$

to yield the following (almost sure) solution:

$$
\begin{aligned}
& \left(\frac{c(\zeta, t)^{1-m / 2}-c\left(\zeta, t_{\mathrm{o}}\right)^{1-m / 2}}{1-\frac{m}{2}}\right) \\
& \quad-m\left(\frac{\pi}{4}\right)^{2}\left(\frac{c(\zeta, t)^{3-m / 2}-c\left(\zeta, t_{\mathrm{o}}\right)^{3-m / 2}}{3-m / 2}\right) \\
& =\int_{t_{\mathrm{o}}}^{t}\left(\Delta S_{\tau}\right)^{m} \Omega_{\tau}\left(\zeta, \Delta S_{\tau}\right) \rho_{\tau}(\zeta) d \tau \quad w p 1 \\
& \quad \text { for } t \geq t_{\mathrm{o}} \text { and } 0<\hat{c}_{t_{\mathrm{o}}}(\zeta) \leq \hat{c}_{t}(\zeta)<\frac{4}{\pi \sqrt{m}}
\end{aligned}
$$

where the constant parameter $m$ is in the range of 2.5 to 5 for ductile alloys and metallic materials ensuring that $(1-m / 2)<0$ and $(3-m / 2)>0$ in Equation (8).

Now we introduce a stochastic diffusion process $\psi_{t}\left(\zeta ; t_{0}\right)$ to represent a (dimensionless) non-negative measure of fatigue crack damage increment from the initial instant $t_{0}$ to the current instant $t$ as a function of the normalized crack lengths $c_{t}(\zeta)$ and $c_{t_{0}}(\zeta)$ of the stressed specimen:

$$
\begin{gathered}
\psi_{t}\left(\zeta ; t_{\mathrm{o}}\right) \equiv\left(\frac{c_{t}(\zeta)^{1-m / 2}-c_{t_{\mathrm{o}}}(\zeta)^{1-m / 2}}{1-m / 2}\right) \\
-m\left(\frac{\pi}{4}\right)^{2}\left(\frac{c_{t}(\zeta)^{3-m / 2}-c_{t_{\mathrm{o}}}^{3-m / 2}}{3-m / 2}\right) w p 1
\end{gathered}
$$

for $t \geq t_{\mathrm{o}}$ and $0<c_{t_{\mathrm{o}}}(\zeta) \leq c_{t}(\zeta)<\frac{4}{\pi \sqrt{m}}$

The damage increment measure is obtained by combining Equations (8) and (9), leading to the following identity in the mean-square $(m s)$ sense:

$$
\psi_{t}\left(\zeta ; t_{\mathrm{o}}\right) \stackrel{m s}{=} \int_{t_{\mathrm{o}}}^{t}\left(\Delta S_{\tau}\right)^{m} \Omega\left(\zeta, \Delta S_{\tau}\right) \rho_{\tau}(\zeta) d \tau
$$

We split the non-negative process $\rho_{\tau}(\zeta)$ in the integrand on the right hand side of Equation (10) as:

$$
\begin{aligned}
\psi_{t}\left(\zeta ; t_{\mathrm{o}}\right) \stackrel{m s}{=} & \int_{t_{\mathrm{o}}}^{t}\left(\Delta S_{\tau}\right)^{m} \Omega\left(\zeta, \Delta S_{\tau}\right) d \tau \\
& +\int_{t_{\mathrm{o}}}^{t}\left(\Delta S_{\tau}\right)^{m} \Omega\left(\zeta, \Delta S_{\tau}\right)\left(\rho_{t}(\zeta)-1\right) d \tau
\end{aligned}
$$

where the zero-mean (possibly correlated) timedependent process $\left(\rho_{t}(\zeta)-1\right)$ is statistically independent of $\Omega\left(\zeta, \Delta S_{t}\right)$ for all $t \geq t_{0}$.

The diffusion process $\psi_{t}\left(\zeta ; t_{0}\right)$ is almost surely continuous because $\psi_{t}\left(\zeta ; t_{0}\right)$ is a continuous function of the crack length process $c_{t}(\zeta) w p 1$. Both $c_{t}(\zeta)$ and $\psi_{t}\left(\zeta ; t_{0}\right)$ are measurable functions although the (probability) measure spaces of $c_{t}(\zeta)$ and $\psi_{t}\left(\zeta ; t_{0}\right)$ are different. In essence, the probability of $\psi_{t}\left(\zeta ; t_{0}\right)$, conditioned on the initial crack length $c_{t_{\mathrm{o}}}(\zeta)$, leads to a stochastic measure of fatigue crack damage increment at the instant $t$ starting from the initial instant $t_{0}$. The conditional probability distribution $F_{\psi \mid c_{t_{0}}}(\cdot ; t \mid \cdot)$ that depends on the history of stress range $\{\Delta S(\tau)$ : $\left.\tau \in\left[t_{\mathrm{o}}, t\right)\right\}$ plays an important role in health monitoring, risk analysis, and remaining life prediction.

Mean and covariance of the stochastic measure of damage increment $\psi_{t}\left(\zeta ; t_{0}\right)$ are expressed as:

$$
\begin{aligned}
\mu_{\psi}\left(t ; t_{\mathrm{o}}\right) \equiv & E\left[\psi_{t}\left(\zeta ; t_{\mathrm{o}}\right)\right] \\
C_{\psi \psi}\left(t_{1}, t_{2} ; t_{\mathrm{o}}\right) \equiv & E\left[\left(\psi_{t_{1}}\left(\zeta ; t_{\mathrm{o}}\right)-\mu_{\psi}\left(t_{1} ; t_{\mathrm{o}}\right)\right)\right. \\
& \left.\times\left(\psi_{t_{2}}\left(\zeta ; t_{\mathrm{o}}\right)-\mu_{\psi}\left(t_{2} ; t_{\mathrm{o}}\right)\right)\right]
\end{aligned}
$$

The covariance function $C_{\psi \psi}\left(t_{1}, t_{2}, t_{3}\right)$ in Equation (12) is continuous at $\left.\left(t_{1}, t_{2}\right)\right|_{t_{1}=t_{2}=t}$ $\forall t \geq t_{\mathrm{o}}$. Hence, the process $\psi_{t}\left(\zeta ; t_{0}\right)$ is mean- 
square $(m s)$ continuous based on a standard theorem of mean-square calculus [13,37].

The objective is to obtain an estimate $\hat{\psi}_{t}\left(\zeta ; t_{\mathrm{o}}\right)$ of the damage increment measure $\psi_{t}\left(\zeta ; t_{0}\right)$ from the initial instant $t_{0}$ to the current instant $t$ such that:

$$
\psi_{t}\left(\zeta ; t_{\mathrm{o}}\right) \stackrel{m s}{=} \hat{\psi}_{t}\left(\zeta ; t_{\mathrm{o}}\right)+\tilde{\psi}_{t}\left(\zeta ; t_{\mathrm{o}}\right)
$$

where the zero-mean estimation error $\tilde{\psi}_{t}\left(\zeta ; t_{\mathrm{o}}\right)$ is statistically orthogonal to $\hat{\psi}_{t}\left(\zeta ; t_{\mathrm{o}}\right)$ in the Hilbert space $L_{2}(P)$ defined by the probability measure $P$ [19]. As such $\hat{\psi}_{t}\left(\zeta ; t_{0}\right)$ is the best linear estimate of $\psi_{t}\left(\zeta ; t_{0}\right)$. Based on mean-square continuity of the damage measure $\psi_{t}\left(\zeta ; t_{0}\right)$, the next section elaborates on the model structure laid out in Equation (13). To this end, we analyze experimental data sets of random fatigue via Karhunen-Loève ( $\mathrm{K}-\mathrm{L})$ decomposition [8,37]. We also use these experimental data sets to identify the model parameters in Section 4.

\section{Karhunen-Loève Decomposition of Experimental Data}

This section analyzes fatigue test data via Karhunen-Loève (K-L) decomposition [8] to justify postulation of the model structure in Equations (8) and (9). We have used experimental data of random fatigue crack growth in 2024-T3 aluminum alloy [35] and 7075-T6 aluminum alloy [9] for which the tests were conducted under different constant load amplitudes at ambient temperature. The Virkler data set was generated for 68 center-cracked specimens (of half-width $w=76.2 \mathrm{~mm})$ at a single constant-amplitude load amplitude with peak nominal stress of $60.33 \mathrm{MPa}$ $(8.75 \mathrm{ksi}), \quad$ stress ratio $R \equiv S_{\min } / S_{\max }=0.2$ for about 200,000 cycles; and the effective stress range $\Delta S^{e}=21.04 \mathrm{MPa}$. The Ghonem data sets were generated for 60 center-cracked specimens each (of half-width $w=50.8 \mathrm{~mm} w=50.8 \mathrm{~mm}$ ) at three different constant load amplitudes: (i) Set 1 with peak nominal stress of $70.65 \mathrm{MPa}(10.25 \mathrm{ksi})$ and $R=0.6$ for 54,000 cycles, the effective stress range $\Delta S^{e}=15.84 \mathrm{MPa}$; (ii) Set 2 with peak nominal stress of $69.00 \mathrm{MPa} \quad(10.00 \mathrm{ksi})$ and
$R=0.5$ for 42,350 cycles, and $\Delta S^{e}=17.80 \mathrm{MPa}$; and (iii) Set 3 with peak nominal stress of $47.09 \mathrm{MPa}$ (6.83 ksi), $R=0.4$ for 73,500 cycles, and $\Delta S^{e}=13.24 \mathrm{MPa}$. The crack opening stress $S^{0}$ is calculated via the correlation of Newman (1984).

For a constant stress range $\Delta S$, Equation (11) is modified as follows:

$$
\begin{aligned}
& \psi_{t}\left(\zeta ; t_{\mathrm{o}}\right) \\
& \stackrel{m s}{=}(\Delta S)^{m} \Omega(\zeta, \Delta S)\left[\left(t-t_{\mathrm{o}}\right)+\theta_{t}\left(\zeta ; t_{\mathrm{o}}\right)\right]
\end{aligned}
$$

where the diffusion $\theta_{t}\left(\zeta ; t_{\mathrm{o}}\right) \equiv \int_{t_{\mathrm{o}}}^{t}\left(\rho_{\tau}(\zeta)-1\right) d \tau$ and $E\left[\theta_{t}\left(\zeta ; t_{\mathrm{o}}\right)\right]=0 \quad \forall t \geq t_{\mathrm{o}}$ because $E\left[\rho_{t}(\zeta)\right]=1$.

Since only finitely many data points at $l$ discrete instants are available from experiments, an obvious choice is discretization over finite time horizons $\left[t_{0}, t\right]$ so that the stochastic process $\psi_{t}\left(\zeta ; t_{0}\right)$ now reduces to an $l$-dimensional random vector denoted as $\psi^{D}(\zeta)$. Consequently, the covariance function $C_{\psi \psi}\left(t_{1}, t_{2}, t_{3}\right)$ in Equation (12) is reduced to a real semi-positive-definite $(l \times l)$ symmetric matrix $C_{\psi \psi}^{D}$. Since the experimental data were collected at sufficiently close intervals, $C_{\psi \psi}^{D}$ contains pertinent information of the crack damage process. The $l$ (real non-negative) eigenvalues of $C_{\psi \psi}^{D}$ are ordered as $\lambda_{1} \geq \lambda_{2} \geq \cdots \geq \lambda_{l}$, with the corresponding eigenvectors, $\varphi^{1}, \varphi^{2}, \ldots, \varphi^{l}$, that form an orthonormal basis of $\mathfrak{R}^{\ell}$ for signal decomposition. The $\mathrm{K}-\mathrm{L}$ decomposition also ensures that the $l$ random coefficients of the basis vectors are statistically orthogonal (i.e., zero-mean and mutually uncorrelated). These random coefficients form a random vector $X(\zeta) \equiv$ $\left[x_{1}(\zeta) x_{2}(\zeta) \ldots x_{l}(\zeta)\right]^{T}$ having the covariance matrix $C_{X X}=\operatorname{diag}\left(\lambda_{1}, \lambda_{2}, \ldots, \lambda_{l}\right)$ leading to decomposition of the discretized signal as:

$$
\psi^{D}(\zeta) \stackrel{m s}{=} E\left[\psi^{D}(\zeta)\right]+\sum_{j=1}^{\ell}\left(\varphi^{j} x_{j}(\zeta)\right)
$$

It was observed by Ray and Tangirala [29] and Ray and Phoha [28] that the statistics of crack length are dominated by the random coefficient corresponding to the principal eigenvector (i.e., the eigenvector associated with the largest eigenvalue) and that the combined effects 
of the remaining eigenvectors are small. Therefore, the signal $\psi^{D}(\zeta)$ in Equation (15) is expressed as the sum of a principal part and a (zero-mean) residual part that are mutually statistically orthogonal:

$$
\psi^{D}(\zeta) \stackrel{m_{s}}{=} \underbrace{E\left[\psi^{D}(\zeta)\right]+\varphi^{1} x_{1}(\zeta)}_{\text {Principal Part }}+\underbrace{\sum_{j=2}^{\ell} \varphi^{j} x_{j}(\zeta)}_{\text {Residual Part }}
$$

The random vector $\psi^{D}(\zeta)$ is expressed as the sum of the principal and residual parts with equality in the mean square $(m s)$ as:

$$
\psi^{D}(\zeta) \stackrel{m s}{=} \hat{\psi}^{D}(\zeta)+\tilde{\psi}^{D}(\zeta)
$$

where $\begin{cases}\hat{\psi}^{D}(\zeta) \equiv E\left[\psi^{D}(\zeta)\right]+\varphi^{1} \chi_{1}(\zeta) & \text { damage estimate } \\ \tilde{\psi}^{D}(\zeta) \equiv \sum_{j=2}^{\ell} \varphi^{j} \chi_{j}(\zeta) & \text { estimation error }\end{cases}$

and the resulting (normalized) mean square error [8] is:

$$
\varepsilon_{r m s}^{2} \equiv \frac{\operatorname{Trace}\left(\operatorname{Cov}\left[\left(\Psi^{D}(\zeta)-\hat{\Psi}^{D}(\zeta)\right)\right]\right)}{\operatorname{Trace}\left(\operatorname{Cov}\left[\Psi^{D}(\zeta)\right]\right)}=\frac{\sum_{j=2}^{\ell} \lambda_{j}}{\sum_{j=1}^{\ell} \lambda_{j}}
$$

The $\mathrm{K}-\mathrm{L}$ decomposition of fatigue test data sets reveals that $\varepsilon_{r m s}^{2}$ in Equation (18) is in the range of $0.018-0.035$ for all four data sets.

The principal eigenvector $\phi^{1}(t)$, associated with the largest eigenvalue $\lambda_{1}$, very closely fits the ramp function $\left(t-t_{0}\right)$ in each experimental data set. Comparing the terms on the right hand side of the discrete model in Equation (17) with those of the continuous model in Equation (14), it is logical to have the random variable $(\Delta S)^{m}$ $\left(\Omega(\zeta, \Delta S)-\mu_{\Omega}(\Delta S)\right)$ to be equal (in $m s$ sense) to the random coefficient $\chi_{1}(\zeta)$ of the principal eigenvector $\phi^{1}(t)$. Applying the lemma in the Appendix, a mean-square equivalence between the $\mathrm{K}-\mathrm{L}$ decomposition model in Equation (16) derived from the test data and the postulated model in Equation (14) is established as:

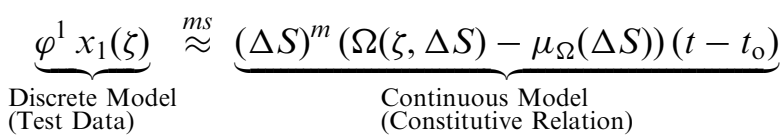

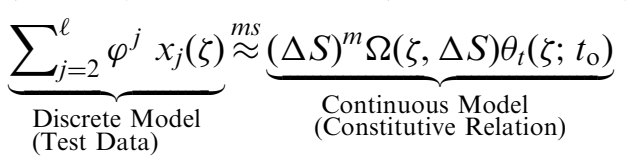

Note that the two entities on the left hand side in Equation (19) are mutually statistically orthogonal. Similarly, the two entities on the right hand side in Equation (19) are also mutually statistically orthogonal. In view of Equation (13), the zero-mean estimation error $\tilde{\psi}_{t}\left(\zeta ; t_{\mathrm{o}}\right)$ is statistically orthogonal to $\hat{\psi}_{t}\left(\zeta ; t_{\mathrm{o}}\right)$ in the Hilbert space $L_{2}(P)$ defined by the probability measure $P$ associated with the stochastic process $\psi_{t}\left(\zeta ; t_{0}\right)$. As such $\hat{\psi}_{t}\left(\zeta ; t_{\mathrm{o}}\right)$ can be viewed as a best linear estimate of $\psi_{t}\left(\zeta ; t_{0}\right)$ with the least error $\tilde{\psi}_{t}\left(\zeta ; t_{\mathrm{o}}\right)$ in the meansquare sense.

It follows from Equations (13) to (19) that the uncertainties associated with an individual sample resulting from the damage measure estimate $\hat{\psi}_{t}\left(\zeta ; t_{\mathrm{o}}\right)$ dominate the cumulative effects of material inhomogeneity and measurement noise in the estimation error $\tilde{\psi}_{t}\left(\zeta ; t_{0}\right)$ unless $\left(t-t_{0}\right)$ is small. Therefore, from the perspectives of health monitoring, risk analysis, and remaining life prediction where the inter-maintenance interval $\left(t-t_{0}\right)$ is expected to be large, a reasonably accurate identification of the mean $\mu_{\Omega}(\Delta S)$ and variance $\sigma_{\Omega}^{2}(\Delta S)$ of the random parameter $\Omega(\zeta ; \Delta S)$ is crucial while the role of the diffusion process $\theta_{t}\left(\zeta ; t_{0}\right)$ is relatively less significant. This observation is consistent with the statistical analysis of fatigue test data by Ditlevsen [7] where the random process described by Equation (19) is treated as the zero-mean residual. Ditlevsen [7] also observed largely similar properties by statistical analysis. Nevertheless, the $\mathrm{K}-\mathrm{L}$ decomposition provides deeper physical insight into the problem.

\section{Model Parameter Identification}

The model parameters, $m, \mu_{\Omega}, \sigma_{\Omega}^{2}$, and $\sigma_{\theta}^{2}$, in Equations (9) and (10) are identified based on the data sets described earlier in Section 3. The 
exponent parameter, $m$, is first identified as an ensemble average estimate from the slope of the logarithm of crack growth rate in Equation (4) for both materials, 7075-T6 and 2024-T3. Following Equation (9), a database for the damage measure $\psi_{t}\left(\zeta ; t_{0}\right)$ is generated for each sample path $\zeta$ from the measured data of crack length $c_{t}(\zeta)$. Since the random parameter $\Omega(\zeta ; \Delta S)$ is not explicitly dependent on time, its expected value is readily identified from Equation (14) as:

$$
\mu_{\Omega}(\Delta S)=\frac{E\left[\psi_{t}\left(\zeta ; t_{\mathrm{o}}\right)\right]}{(\Delta S)^{m}\left(t-t_{\mathrm{o}}\right)}
$$

from the ensemble average estimate from the data sets for each type of material, 7075-T6 and 2024T3 alloys.

Data analysis of individual sample paths in fatigue crack propagation reveals that the stochastic process $\rho_{t}(\zeta)$ in Equation (10) is positively correlated. Therefore, we postulate that the zeromean diffusion process $\theta_{t}\left(\zeta ; t_{\mathrm{o}}\right) \equiv \int_{t_{\mathrm{o}}}^{t}\left(\rho_{\tau}(\zeta)-1\right) d \tau$ is a (positively correlated) fractional Brownian motion $(\mathrm{fBm})$ process $[18,36]$ such that

$$
\operatorname{Var}\left[\theta_{t}\left(\zeta ; t_{\mathrm{o}}\right)\right] \equiv \sigma_{\theta}^{2}\left(t ; t_{\mathrm{o}}\right)=\Phi\left(t-t_{\mathrm{o}}\right)^{\alpha}
$$

where the exponent $1<\alpha<2$ represents positive correlation.

Following Equation (14), the variance $\sigma_{\psi}^{2}\left(t, t_{\mathrm{o}}\right)$ of the damage increment measure $\left.\psi_{t}\left(\zeta ; t_{0}\right)\right)$ is obtained from experimental data as:

$$
\begin{aligned}
& \sigma_{\psi}^{2}\left(t ; t_{\mathrm{o}}\right) \\
& \quad=(\Delta S)^{2 m}\left(\sigma_{\Omega}^{2}\left(t-t_{\mathrm{o}}\right)^{2}+\left(\sigma_{\Omega}^{2}+\mu_{\Omega}^{2}\right) \sigma_{\theta}^{2}\left(t ; t_{\mathrm{o}}\right)\right)
\end{aligned}
$$

However, since the variances $\sigma_{\Omega}^{2}(\Delta S)$ and $\sigma_{\theta}^{2}\left(t ; t_{\mathrm{o}}\right)$ of $\Omega(\zeta ; \Delta S)$ and $\theta_{t}\left(\zeta ; t_{0}\right)$, respectively, cannot be separately identified from Equation (22) alone, we make use of the eigenvalues, $\lambda_{1}$, $\lambda_{2}, \ldots, \lambda_{l}$, of $C_{\psi \psi}^{D}$ generated by $\mathrm{K}-\mathrm{L}$ decomposition as additional information. Taking expected values of the Euclidean norms on both sides of the pair of Equations (19) and using Equation (17), the following relations are obtained based on the test data over a period $\left[t_{0}, t\right]$ :

$$
\begin{gathered}
(\Delta S)^{2 m} \sigma_{\Omega}^{2}(\Delta S)\left(t-t_{\mathrm{o}}\right)^{2} \approx \lambda_{1}\left(t, t_{\mathrm{o}}\right) \\
\Rightarrow \quad \sigma_{\Omega}^{2}(\Delta S) \approx \frac{\lambda_{1}\left(t, t_{\mathrm{o}}\right)}{(\Delta S)^{2 m}\left(t-t_{\mathrm{o}}\right)^{2}} \\
(\Delta S)^{2 m} \operatorname{Var}\left[\Omega(\zeta, \Delta S) \theta_{t}\left(\zeta ; t_{\mathrm{o}}\right)\right] \approx \sum_{j=2}^{\ell} \lambda_{j}\left(t, t_{\mathrm{o}}\right) \\
\Rightarrow \quad \Phi\left(t-t_{\mathrm{o}}\right)^{\alpha} \approx \frac{\sum_{j=2}^{\ell} \lambda_{j}\left(t, t_{\mathrm{o}}\right)}{(\Delta S)^{2 m}\left(\sigma_{\Omega}^{2}+\mu_{\Omega}^{2}\right)}
\end{gathered}
$$

Substitution of the parameters $\mu_{\Omega}(\Delta S)$ and $\sigma_{\Omega}^{2}(\Delta S)$ from Equations (20) and (23) in Equation (24) yields:

$$
\Phi\left(t-t_{\mathrm{o}}\right)^{\alpha-2} \approx \frac{\sum_{j=2}^{\ell} \lambda_{j}\left(t, t_{\mathrm{o}}\right)}{\lambda_{1}\left(t, t_{\mathrm{o}}\right)+E^{2}\left[\psi\left(\zeta, t ; t_{\mathrm{o}}\right)\right]}
$$

The unknown parameters, $\alpha$ and $\Phi$, on the left hand side of Equation (25) are now identified by linear regression analysis of experimentally derived data on the right hand side of Equation (25) for different ranges of $t$. The value of $\alpha$ is found to be $\sim 1.3$, which signifies that the diffusion $\theta_{t}\left(\zeta ; t_{0}\right)$ associated with the estimation error $\tilde{\psi}$, can be approximated as a (positively correlated) fractional Brownian motion.

The following generalized parametric relations are now postulated for different levels of (constant-amplitude) stress excitation $\Delta S$ for a given material:

- $\mu_{\Omega}(\Delta S) \equiv E[\Omega(\zeta, \Delta S)]$ is independent of $\Delta S$, i.e., $\mu_{\Omega}$ is a constant for a given material and $E\left[(\Delta S)^{m} \Omega(\zeta, \Delta S)\right]=(\Delta S)^{m} \mu_{\Omega}$.

- $\sigma_{\Omega}^{2}(\Delta S) \equiv \operatorname{Var}[\Omega(\zeta, \Delta S)]$ is proportional to $(\Delta S)^{-2 m}$, i.e., $\operatorname{Var}\left[(\Delta S)^{m} \Omega(\zeta, \Delta S)\right]$ is a constant for a given material.

- $\operatorname{Var}\left[(\Delta S)^{m} \Omega(\zeta) \theta_{t}\left(\zeta ; t_{\mathrm{o}}\right)\right] \approx \sigma_{\Omega}^{2}(\Delta S) \Phi\left(t-t_{\mathrm{o}}\right)^{\alpha}$ becomes gradually small compared to $\operatorname{Var}\left[(\Delta S)^{m} \Omega(\zeta)\right]\left(t-t_{\mathrm{o}}\right)^{2}$ for large $\left(t-t_{0}\right)$ because the variance of the fractional Brownian motion $\theta_{t}\left(\zeta, t_{0}\right)$ has an exponent $\alpha<2$. 
The above three observations are consistent with the data sets of Ghonem and Dore [9] for 7075-T6 aluminum alloy. The first two observations are not yet verified for 2024-T3 aluminum alloy because the Virkler data set provides only one level of stress range. These relations are expected to be valid for ductile alloys and many other metallic materials because the nature of dependence of the model parameters on the material microstructure and specimen preparation (i.e., machining operations) is similar. Laboratory experiments are planned to generate additional test data and significant results will be reported in forthcoming publications.

Several investigators have assumed the crack growth rate in ductile alloys to be lognormaldistributed (e.g., citations in Sobczyk and Spencer [31]). Some others have treated the crack length to be lognormal-distributed (e.g., Ray and Tangirala [29]). The results of $\mathrm{K}-\mathrm{L}$ decomposition in Equations (12) to (17) are in agreement with these claims because $\Omega(\zeta, \Delta S)$ which dominates the random behavior of fatigue crack growth can be considered as a perfectly correlated random process whereas the non-negative, multiplicative uncertainty term $\rho_{t}(\zeta)$ is a weakly (positively) correlated random process. Yang and Manning [38] have presented an empirical second-order approximation of crack growth by postulating lognormal distribution of a parameter that does not bear any physical relationship to $\Delta S$ but it is, to some extent, similar to $\Omega(\zeta, \Delta S)$ in the present model.

We hypothesize that the random process $\Omega(\zeta, \Delta S)$ is two-parameter $(r=2)$ lognormaldistributed [5], and its goodness of fit is examined by both $\chi^{2}$ and Kolmogorov-Smirnov tests of experimental data. Each of the four data sets is partitioned into $L=12$ segments to assure that each segment contains at least 5 samples. With $(L-r-1)=9$ degrees of freedom, the $\chi^{2}$-test shows that, for each of the four data sets, the hypothesis of two-parameter lognormal-distribution of $\Omega(\zeta, \Delta S)$ passed the $10 \%$ significance level which suffices the conventional standard of $5 \%$ significance level. For each of the four data sets, the hypothesis of two-parameter lognormal-distribution of $\Omega(\zeta, \Delta S)$ also passed the $20 \%$ significance level of the Kolmogorov-Smirnov test. The probability density function (pdf) of the twoparameter lognormal-distributed random variable $\Omega(\zeta, \Delta S)$ is obtained for a given value of $\Delta S$ as [5]:

$$
f_{\Omega}(x)=\left\{\begin{array}{cc}
\frac{\exp \left(-\frac{(\ln x-\mu)^{2}}{2 \sigma^{2}}\right)}{\sigma x \sqrt{2 \pi}} & \text { for } x \geq 0 \\
0 & \text { otherwise }
\end{array}\right.
$$

where $\sigma^{2} \equiv \log \left(1+\left(\sigma_{\Omega} / \mu_{\Omega}\right)^{2}\right)$ and $\mu \equiv \log \left(\mu_{\Omega}\right)-$ $\sigma^{2} / 2$.

Now we summarize the results of model parameter identification. The random process $\Omega(\zeta, \Delta S)$ is hypothesized as two-parameter $(r=2)$ lognormal-distributed [5], and its goodness of fit is examined by both $\chi^{2}$ and KolmogorovSmirnov tests [3] on the experimental data. Each of the four data sets is partitioned into $L=12$ segments to assure that each segment contains at least 5 samples. With $(L-r-1)=9$ degrees of freedom, the $\chi^{2}$-test shows that, for each of the four data sets, the hypothesis of two-parameter lognormal-distribution of $\Omega(\zeta, \Delta S)$ passed the $10 \%$ significance level which suffices the conventional standard of $5 \%$ significance level. For each of the four data sets, the hypothesis of twoparameter lognormal-distribution of $\Omega(\zeta, \Delta S)$ also passed the $20 \%$ significance level of the Kolmogorov-Smirnov test.

Figure 1 compares the analytically derived lognormal-distributed probability density functions (pdf's) of $\Omega(\zeta, \Delta S)$ with the corresponding histograms generated from four sets of experimental data by compensating the relatively small second-order statistics of the diffusion $\theta_{t}\left(\zeta ; t_{0}\right)$ as delineated in Equation (24). The mean $\mu_{\Omega}$ in the model is identical for the three data sets of 7075-T6 while the corresponding variance is different in each set as seen in Figure 1. This is because $\sigma_{\Omega}^{2}(\Delta S)$ is inversely proportional to $(\Delta S)^{2 \mathrm{~m}}$ and $\Delta S$ is different for each data set $\sigma_{\Omega}^{2}$ is largest for the Ghonem data set \#3 for which $\Delta S^{e}=13.24 \mathrm{MPa}$ is smallest and $\sigma_{\Omega}^{2}$ is smallest for the Ghonem data set \#2 for which $\Delta S^{e}=17.80 \mathrm{MPa}$ is largest of the three data sets. However, for 2024-T3, no such comparison could be made because only one $\Delta S^{e}$ is available in the Virkler data set. 

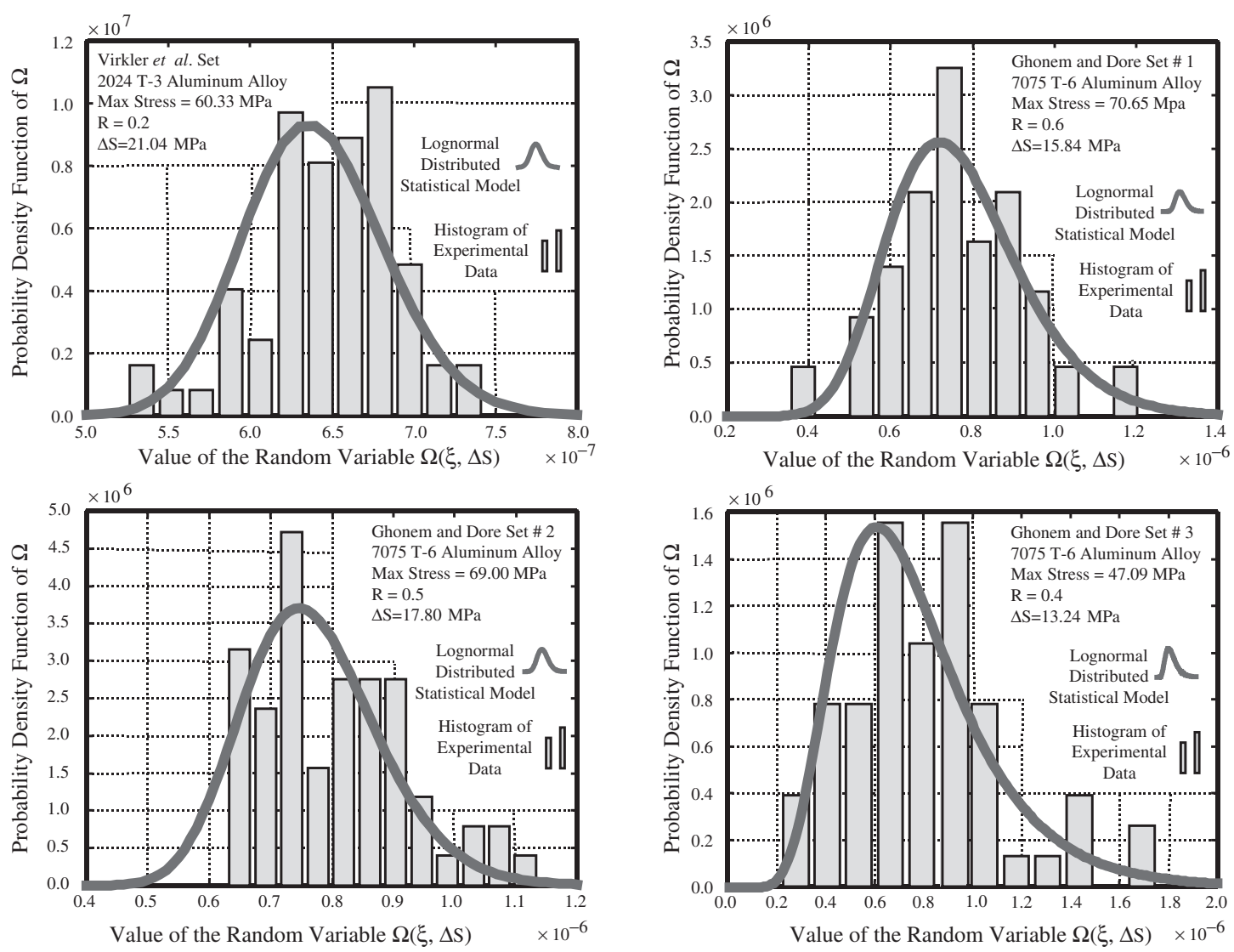

Figure 1 Identification of probability density function of the model parameter $\Omega$.

We have not made any formal hypothesis on the probability distribution of $\rho_{t}(\zeta)$ because the zero-mean diffusion $\theta_{t}\left(\zeta ; t_{\mathrm{o}}\right) \equiv \int_{t_{\mathrm{o}}}^{t}\left(\rho_{\tau}(\zeta)-1\right) d \tau$ has been approximated as a fractional Brownian motion.

\section{Model Prediction}

Model predictions of crack growth are now obtained by Monte Carlo simulation of the stochastic difference equation (5). Lognormal distributions of $\Omega(\zeta, \Delta S)$ are realized by taking exponentials of outputs of the standard normal random number generator with different seed numbers. For convenience of simulation, we have represented the approximate fractional Brownian motion process $\theta_{t}\left(\zeta ; t_{0}\right)$ as a zero-mean Gaussian distribution $N\left(0, \Phi\left(t-t_{0}\right)^{\alpha}\right)$. It should be noted that the parameters $\Phi$ and $\alpha$ are quite approximate because of a very small contribution of the estimation error term $\tilde{\psi}$ relative to the estimate $\hat{\psi}$ as seen in Equation (18). Figure 2 shows a comparison of the test data of fatigue crack growth and the predictions of Monte Carlo simulation of the damage model in Equation (5) for the four data sets under consideration.

Next we present the accuracy of probability distribution functions (PDFs) of the estimated damage measure $\hat{\psi}$, which are generated from the lognormal-distributed parameter $\Omega(\zeta, \Delta S)$ from Equations (12) and (23). Both test data and model predictions are used to generate PDFs of service cycles to exceed specified limits $a^{*}$ of crack length. Note that the Virkler set and each of the three Ghonem sets contain 68 samples and 60 samples, respectively, while the Monte Carlo simulations for model prediction have been conducted with 1000 samples in each case. The PDF plots in Figure 3 compare model predictions with the experimental data of Virkler 

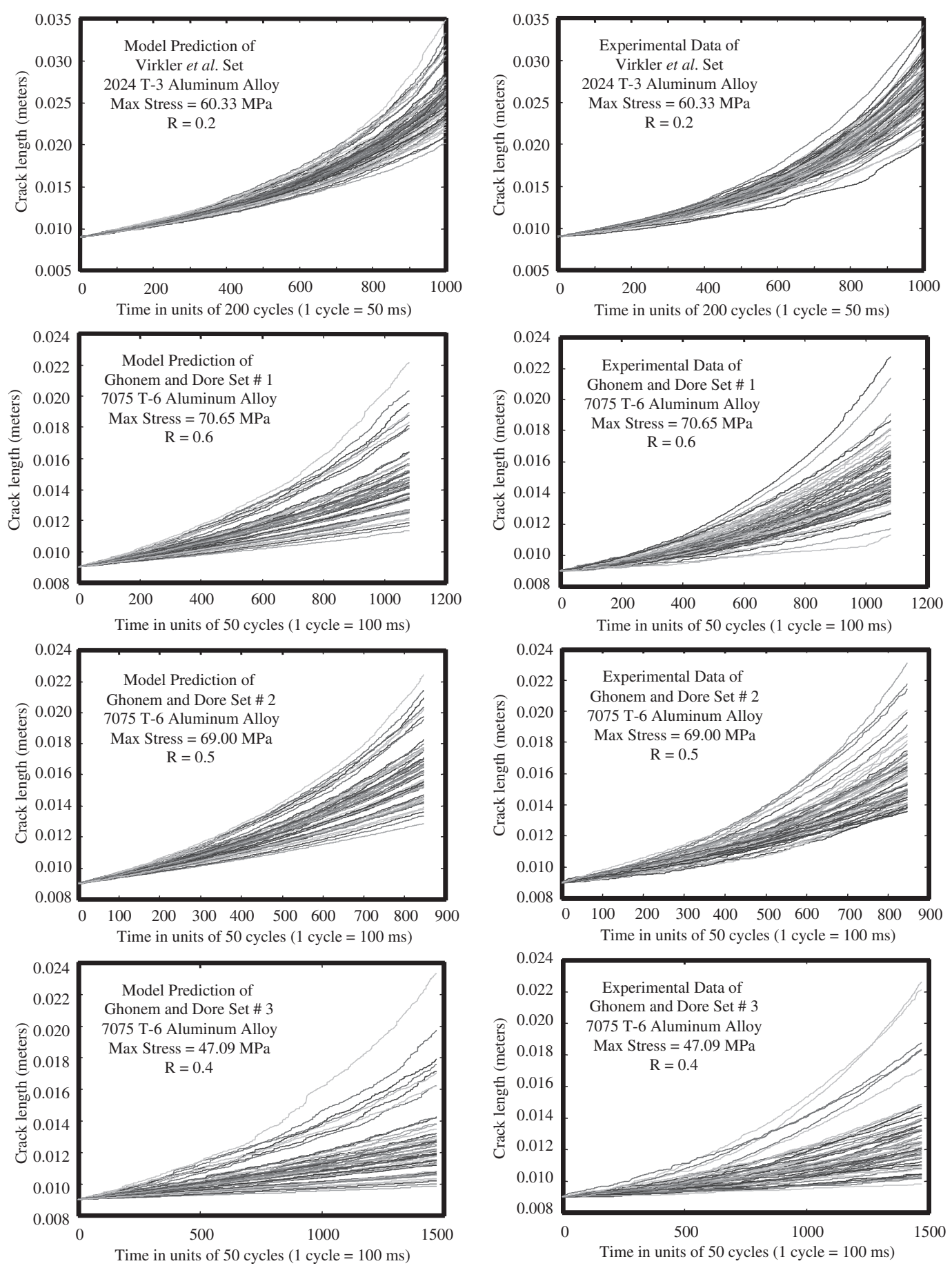

Figure 2 Comparison of model prediction with experimental data for 2024-T3 and 7075-T6.

et al. (1979) for three different values of $a^{*}$ at $11 \mathrm{~mm}, 14 \mathrm{~mm}$, and $20 \mathrm{~mm}$. Similarly, the three PDF plots from left to right in Figure 4 compare model predictions with the data sets, $\# 2$, \#1, and \#3 (in the decreasing order of the effective stress range $\Delta S^{e}$ ), respectively, of Ghonem and Dore [9] data sets at $a^{*}=11 \mathrm{~mm}$. The agreement of the predicted PDFs in Figures 3 and 4 with the respective experimental data is a consequence of fitting the key model parameter $\Omega(\zeta, \Delta S)$ to a 


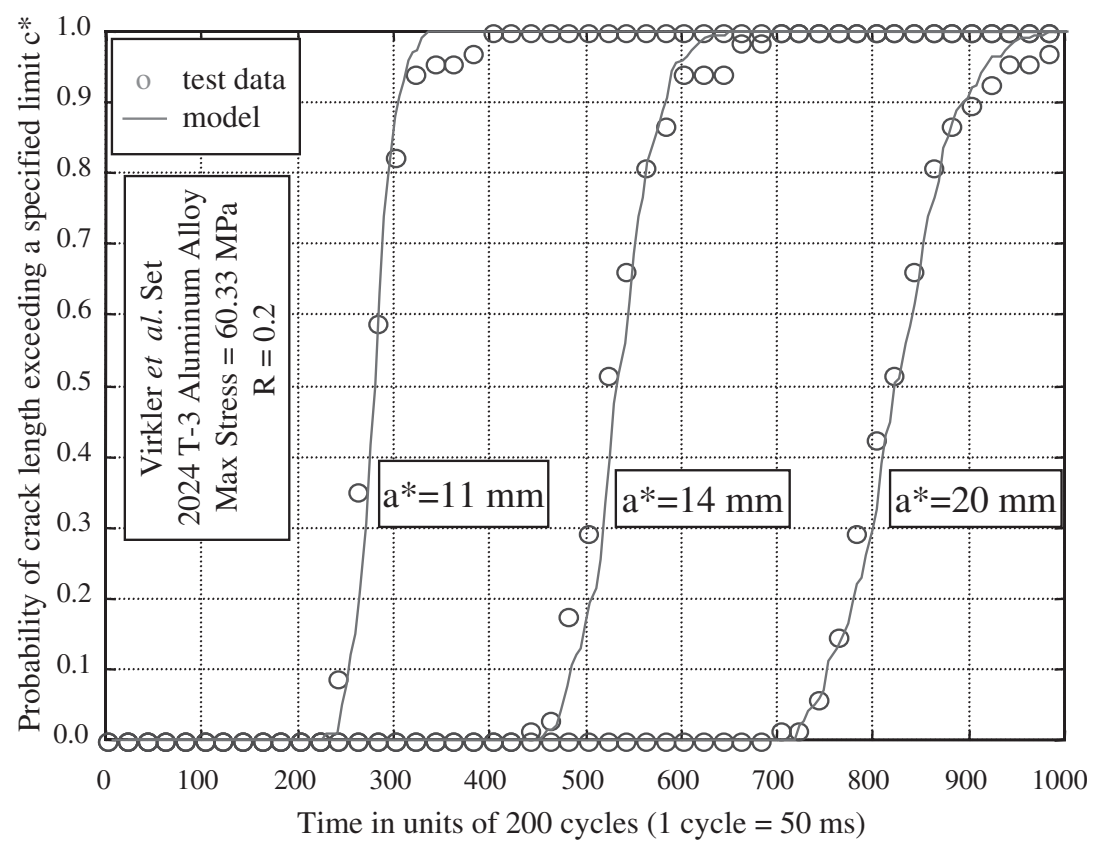

Figure 3 Probability distribution of crack length exceeding specified limits (Virkler et al. Data).

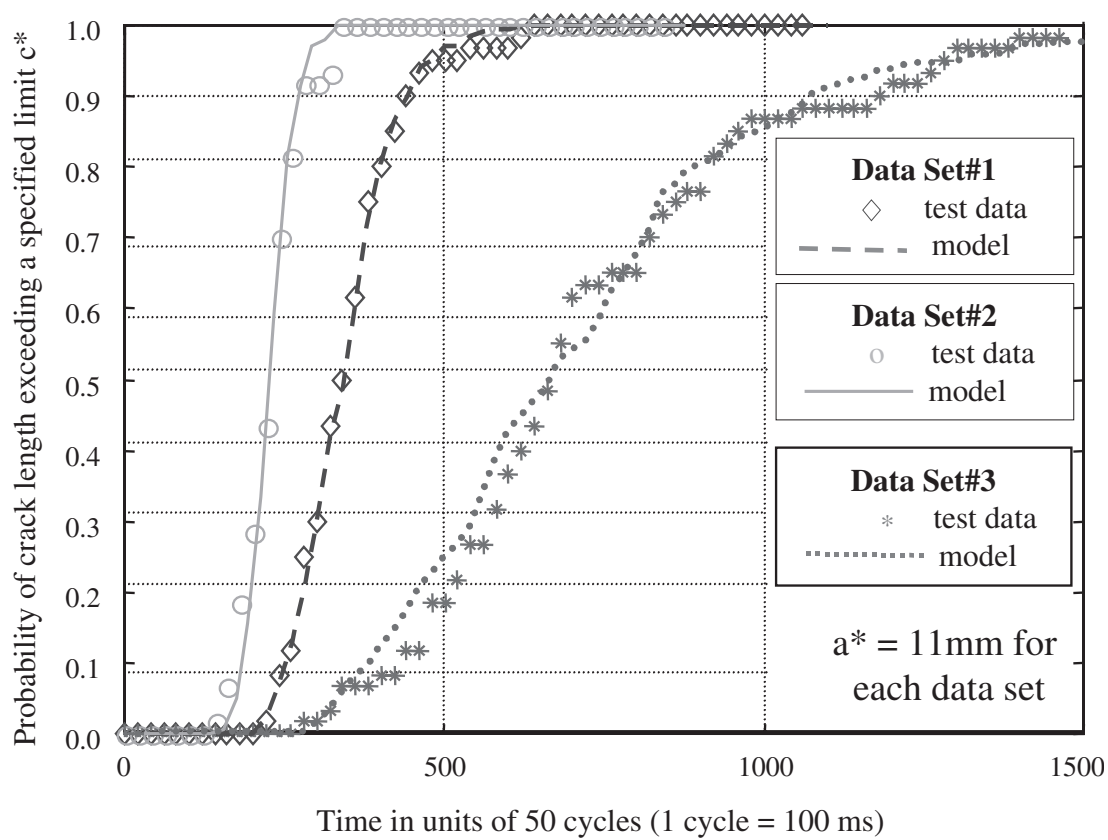

Figure 4 Probability distribution of crack length exceeding a specified limit (Ghonem \& Dore Data).

high level of statistical significance as seen in Figure 1. The small differences between the modelbased and experimental PDFs in both Figures 3 and 4 should be further reduced for larger ensemble size of the data sets as the histograms of $\Omega(\zeta, \Delta S)$ in Figure 1 would more closely fit the (right hand) tails of the probability density function (pdf) plots. 


\section{Risk Analysis and Life Prediction}

This section illustrates how the stochastic model of fatigue crack growth, developed in earlier sections, can be used for risk analysis and remaining life prediction of mechanical structures. As pointed out earlier, the impact of $\rho_{t}(\zeta)$ and hence of the diffusion $\theta_{t}\left(\zeta ; t_{0}\right)$ on overall scatter of the crack growth profile is not significant for large $\left(t-t_{0}\right)$. In general, $t_{0}$ signifies the starting time of a machine after maintenance and/or inspection. Since risk analysis and life prediction become important after a significant lapse of time (i.e., when $\left(t-t_{0}\right)$ is sufficiently large), it is reasonable to make these decisions based only on the PDF of the estimated damage measure $\hat{\psi}_{t}\left(\zeta ; t_{\mathrm{o}}\right)$. Note that the error due to ignoring the effects of the error $\tilde{\psi}_{t}\left(\zeta ; t_{0}\right)$ of damage measure estimation is on the order of $\sum_{j=2}^{\ell} \lambda j / \sum_{j=1}^{\ell} \lambda j$ that is in the range of 0.018 to 0.035 for all four sets as stated in Section 2.

\subsection{Hypotheses Testing for Risk Analysis}

This subsection presents a simple example to demonstrate how the estimate $\hat{\psi}_{t}\left(\zeta ; t_{\mathrm{o}}\right)$ of stochastic damage measure can be used for hypothesis testing for risk analysis.

Let $(M+1)$ levels of hypotheses be defined based on a partition of the physical variable, crack length, in the range $\left[\bar{c}_{0}, \infty\right)$ where $\bar{c}_{o}$ is the (a priori known) minimum threshold of the initial crack length $c\left(\zeta, t_{0}\right)$ which is assumed to be measured with good precision, i.e., $\sigma_{\bar{c}_{0}}^{2} \approx 0$. The parameter $\bar{c}_{o}$ represents an estimate of the effects of initial crack or defect based on the available information from sensing (e.g., ultrasonic, electromagnetic, mechanical, or optical) devices.

The first $M$ hypotheses are defined on the range $\left[\bar{c}_{\mathrm{o}}, \bar{c}_{M}\right]$ where $\bar{c}_{M}$ is the critical crack length beyond which the crack growth rate rapidly becomes very large leading to complete rupture:

$$
\begin{array}{ccrl}
H_{0}\left(t, t_{\mathrm{o}}\right): & & c(\zeta, t) & \in\left[\bar{c}_{o}, \bar{c}_{1}\right) \\
H_{1}\left(t, t_{\mathrm{o}}\right): & & c(\zeta, t) & \in\left[\bar{c}_{1}, \bar{c}_{2}\right) \\
\vdots & & \vdots & \\
H_{M-1}\left(t, t_{\mathrm{o}}\right): & & c(\zeta, t) & \in\left[\bar{c}_{M-1}, \bar{c}_{M}\right)
\end{array}
$$

where $\bar{c}_{i}=\bar{c}_{0}+i\left(\bar{c}_{M}-\bar{c}_{0}\right) / M, i=1,2, \ldots,(M-1)$.

The last (i.e., the $M$ th) hypothesis is defined as $H_{M}: c_{\tau} \in\left[\bar{c}_{M}, \infty\right)$, which is popularly known as the unstable crack region in the fracture mechanics literature [31]. Each of these $(M+1)$ hypotheses represents a distinct range in the entire space of crack lengths from an initial value till rupture occurs, and together, they form an exhaustive set of mutually exclusive regions in the state space of crack length. The first $M$ hypotheses are generated as:

$$
\begin{gathered}
c_{t}(\zeta) \in H_{j}\left(t, t_{\mathrm{o}}\right)=\left[\bar{c}_{j}, \bar{c}_{j+1}\right) \\
\Rightarrow \psi_{t}\left(\zeta ; t_{\mathrm{o}}\right) \in\left[\psi_{j}, \psi_{j+1}\right)
\end{gathered}
$$

for $j=0,1,2, \ldots, M-1$ and a given $\Delta S$.

$$
\text { where } \begin{aligned}
\psi_{j} & \equiv \frac{\left(\bar{c}_{j} / w\right)^{1-m / 2}-\left(\bar{c}_{\mathrm{o}} / w\right)^{1-m / 2}}{1-m / 2} \\
& -m\left(\frac{\pi}{4}\right)^{2}\left(\frac{\left(\bar{c}_{j} / w\right)^{3-m / 2}-\left(\bar{c}_{\mathrm{o}} / w\right)^{3-m / 2}}{3-m / 2}\right)
\end{aligned}
$$

follows the structure of Equation (7). As discussed earlier, the damage measure $\psi_{t}\left(\zeta ; t_{0}\right)$ is approximated as the estimate $\hat{\psi}_{t}\left(\zeta ; t_{\mathrm{o}}\right)$ by ignoring the effects of the error term $\tilde{\psi}_{t}\left(\zeta ; t_{0}\right)$. The probability that the $j$ th hypothesis, $H_{j}\left(t, t_{0}\right)$, can be obtained from the instantaneous (conditional) PDF $F_{\psi \mid \bar{c}_{t_{0}}}(\cdot ; t)$ of $\psi\left(\zeta, t ; t_{0}\right)$. This is directly generated from the two-parameter lognormal distribution of $\Omega(\zeta, \Delta S)$ without any computationally expensive integration because conversion of the range of integration in the log scale allows evaluation of the error function via tablelookup. These details are straight-forward and are not presented in this paper.

For $j=0,1,2, \ldots, M-1$, the probabilities of the individual hypotheses become:

$$
\begin{aligned}
& P\left[H_{j}\left(t, t_{\mathrm{o}}\right)\right] \\
& \quad=F_{\psi \mid c\left(\zeta, t_{\mathrm{o}}\right)}\left(\psi_{j+1} ;\left.t\right|_{\bar{c}_{\mathrm{o}}}\right)-F_{\psi \mid c\left(\zeta, t_{\mathrm{o}}\right)}\left(\psi_{j} ;\left.t\right|_{\bar{c}_{\mathrm{o}}}\right)
\end{aligned}
$$

and 


$$
P\left[H_{M}\left(t, t_{\mathrm{o}}\right)\right]=1-\sum_{j=0}^{M-1} P\left[H_{j}\left(t, t_{\mathrm{o}}\right)\right]
$$

The concept of hypothesis testing for risk analysis and life prediction is now elucidated with Virkler and Ghonem data sets. The probability that the random crack length $\left\{c(\zeta, \mathrm{t}): t \geq t_{0}\right\}$ at a given time $t$ is located in one and only one of these segments is computed in real time by Equation (24). For each data set, it is observed that $\bar{a}_{\mathrm{o}}=9.0 \mathrm{~mm}$, i.e., $\bar{c}_{\mathrm{o}}=\bar{a}_{\mathrm{o}} / w, w p 1$. The critical crack length, $\bar{c}_{M}=\bar{a}_{M} / w$, is chosen based on the geometry of the test specimens: For the Virkler experiment (in which the specimen half-width is $76.2 \mathrm{~mm}), \bar{a}_{M}=45.0 \mathrm{~mm}$; for the Ghonem experiments (in which the specimen half-width is $50.4 \mathrm{~mm}), \quad \bar{a}_{M}=27.0 \mathrm{~mm}$. The space $\left[\bar{c}_{o}, \infty\right)$ is partitioned into $(M+1)$ regions. In these examples, we have chosen 11 hypotheses (i.e., $M=10$ ) for both Virkler and Ghonem data sets. The range of each hypothesis is defined as depicted in Table I and Table II, respectively. The time evolution of probability of the hypotheses for the four data sets is shown in the four plates of Figure 5. In each case, the plot of $H_{0}$ begins with a probability equal to 1 at time $t=t_{0}$ and later diminishes as the crack grows with time (i.e., number of load cycles applied). The probability of each of the hypotheses $H_{1}$ to $H_{9}$ is initially zero and then increases to a maximum and subsequently decreases as the crack growth process progresses with time. The probability of the last hypothesis $H_{10}$ (on the extreme right in each plate of Figure 5) of unstable crack growth beyond the critical crack length, $\bar{c}_{M}$, initially remains at zero and increases rapidly only when the specimen is close to rupture. At this stage, the probability of each of the remaining hypotheses is zero or rapidly diminishes to zero.

The hypotheses testing procedure can be executed in real time on inexpensive platforms such as a Pentium processor in the plant instrumentation and control system for issuing alerts and warnings while the machine is in operation. For example, the space of crack length, defined by $\left[\bar{c}_{0}, \infty\right)$, can be partitioned into four hypotheses denoting three regions of green, yellow, and red alert conditions for the first three
Table 1 Crack damage hypotheses for Virkler et al. Data.

\begin{tabular}{ll}
\hline Description & Range of Fatigue Crack Length \\
\hline Hypothesis $\mathrm{H}_{0}$ & $9.00 \mathrm{~mm} \leq \mathrm{a}(t)<12.6 \mathrm{~mm}$ \\
Hypothesis $\mathrm{H}_{1}$ & $12.6 \mathrm{~mm} \leq \mathrm{a}(t)<16.2 \mathrm{~mm}$ \\
$\cdot$ & $\cdot$ \\
$\cdot$ & $\cdot$ \\
$\cdot$ & $\cdot$ \\
Hypothesis $\mathrm{H}_{9}$ & $41.4 \mathrm{~mm} \leq \mathrm{a}(t)<45.0 \mathrm{~mm}$ \\
Hypothesis $\mathrm{H}_{10}$ & $45.0 \mathrm{~mm} \leq \mathrm{a}(t)$ \\
& (Unstable crack growth) \\
\hline
\end{tabular}

Table 2 Crack damage hypotheses for Ghonem \& Dore Data.

\begin{tabular}{ll}
\hline Description & Range of Fatigue Crack Length \\
\hline Hypothesis $\mathrm{H}_{0}$ & $9.00 \mathrm{~mm} \leq \mathrm{a}(t)<10.8 \mathrm{~mm}$ \\
Hypothesis $\mathrm{H}_{1}$ & $10.8 \mathrm{~mm} \leq \mathrm{a}(t)<12.6 \mathrm{~mm}$
\end{tabular}

Hypothesis $\mathrm{H}_{9} \quad 25.2 \mathrm{~mm} \leq \mathrm{a}(t)<27.0 \mathrm{~mm}$ Hypothesis $\mathrm{H}_{10} \quad 27.0 \mathrm{~mm} \leq \mathrm{a}(t)$ (Unstable crack growth)

hypotheses and catastrophic conditions for the fourth hypothesis. While alerts and warnings are useful for operational support and safety enhancement, operations planning and maintenance scheduling require remaining life prediction. Equipment readiness assessment and failure prognosis based on current condition and projected usage of the machinery are important tools for operations and maintenance planning, especially in an information-based maintenance environment where access to all pertinent information is enabled.

\subsection{Remaining Life Prediction}

Having known the instantaneous (conditional) probability distribution function $F_{\hat{\psi} \mid c_{0}}\left(\cdot ;\left.t\right|_{\bar{c}_{0}}\right)$ of the estimated damage $\hat{\psi}_{t}\left(\zeta ; t_{0}\right)$, the remaining life can be computed on-line at any specified time instant, $t$, based on a desired plant operational profile and a confidence level $(1-\varepsilon)$. This implies that if the plant operation is scheduled to yield a desired output profile, then the remaining life $T$ is the maximum time of operation after the 

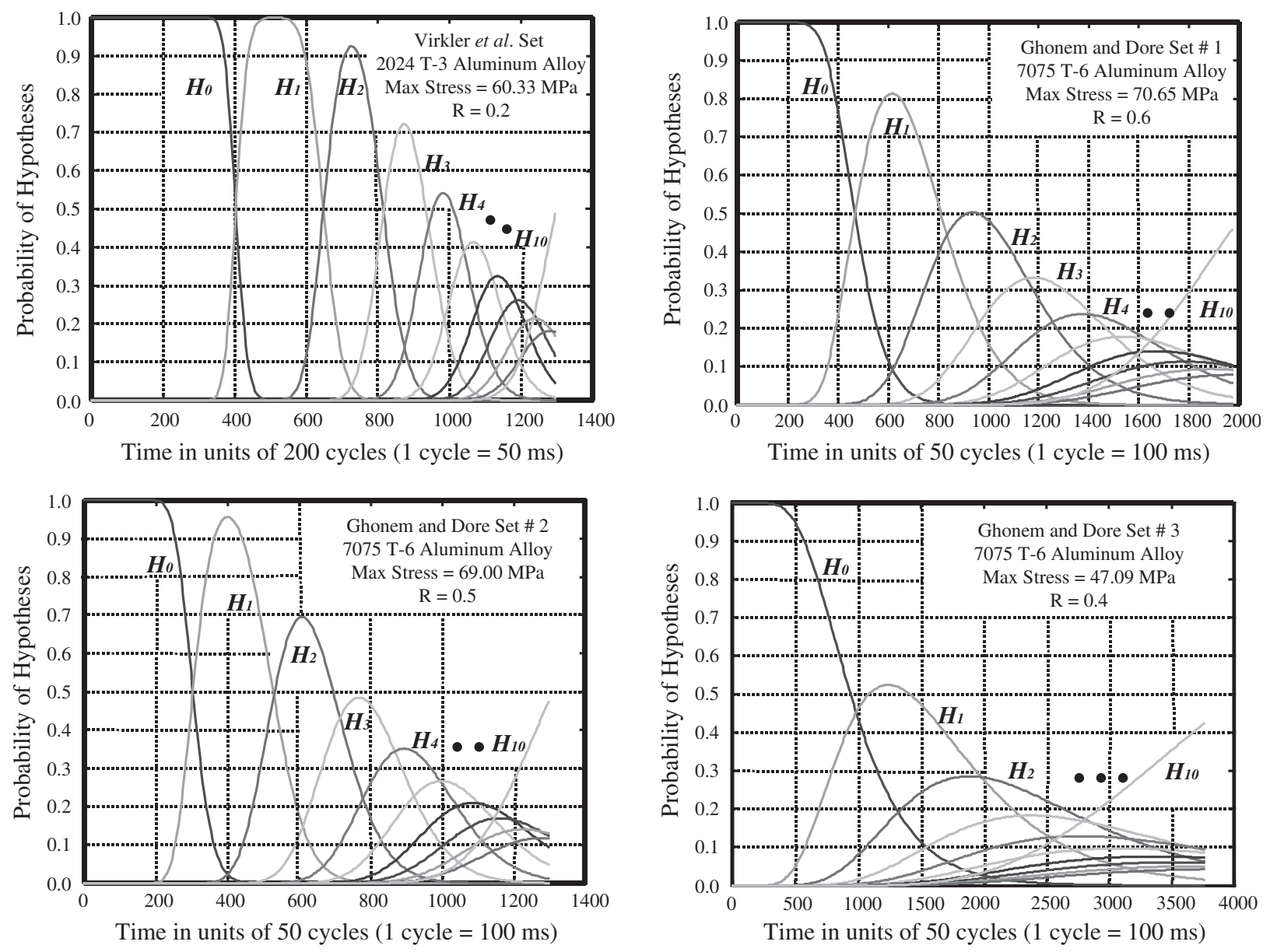

Figure 5 Probabilities of hypotheses for fatigue crack propagation.

current time such that the probability of the crack length $c_{t+\Gamma}(\zeta)$ to exceed a specified bound $\bar{c}_{M}$ is less than $\varepsilon$.

The algorithm for remaining life prediction must be executed in real time based on the current information. The generated results can then be conveyed to a decision-making module at a higher level for failure prognosis, life extending control, and maintenance scheduling, or simply for generation of warnings and alerts. These results may also be displayed as a decision support tool for human operators. The objective is to determine the statistical confidence with which plant operations can be planned for a specified period of time or to evaluate alternate operational scenarios. This is also of considerable importance in the scheduling of maintenance to avoid untimely shutdowns since failure prognostic information is inherent in remaining life prediction. Some of these issues have been addressed by Ray and Phoha [28].

\section{Summary and Conclusions}

This paper presents a stochastic measure estimate of fatigue crack damage for health monitoring, risk analysis, and life prediction of ductile alloy structures and machinery components in mechanical systems (e.g., aircraft, spacecraft, and power plants). The constitutive equation of the damage measure is based on the physics of fracture mechanics. The stochastic damage measure model is built upon the state-space model of fatigue crack growth [27], which predicts an estimated mean of crack length and accounts for variableamplitude cyclic stress and crack retardation 
phenomena. The model of stochastic damage measure model is potentially applicable to health monitoring of mechanical structures that might be subjected to variable-amplitude stress cycling. However, the model parameters (that depend on the structural material and crack geometry) have to be identified and tuned a priori in the laboratory environment [14].

The damage measure is modeled as a diffusion process represented by a continuous function of the current crack length and the initial crack length. The randomness in the damage measure estimate accrues from manufacturing uncertainties such as defects generated during machining operations and is captured by a single lognormaldistributed non-stationary random variable. The resulting diffusion process of estimation error is primarily due to inhomogeneity in the structural material, and is approximated by a fractional Brownian motion (fBm) model. The damage estimate is statistically orthogonal to the resulting zero-mean estimation error in the Hilbert space $L_{2}(P)$ defined by the probability measure $P$ of the stochastic damage. As such the damage estimate can be viewed as a best linear estimate with least error in the mean-square sense.

The structure of the stochastic damage model has been validated by Karhunen-Loève decomposition of fatigue test data in the Hilbert space setting for 2024-T3 at a single level of constantamplitude cyclic load and for 7075-T6 aluminum alloys at three different levels of constant-amplitude cyclic load. For 7075-T6 alloy, predictions of the stochastic damage model, identified and tuned for the experimental data set under one load condition, closely matches those under two other load conditions. For 2024-T3 alloy, the same model structure prevails. This establishes predictive capability of the stochastic damage model under constant-amplitude loading. Validation of the stochastic damage model under varying-amplitude random loading is a subject of current research as an extension of the author's earlier work [26].

A systematic procedure for parameter identification of the stochastic damage measure model has been established. The predicted probability distribution function (PDF) of service cycles to exceed a specified crack length is shown to be in close agreement with those generated from a number of test data sets. The (non-stationary) probability distribution function of crack damage measure is obtained in a closed form without numerically solving stochastic differential equations in the Wiener integral or Itô integral setting $[13,22,37]$. The model allows formulation of risk assessment and life prediction algorithms for real-time execution on inexpensive platforms such as a Pentium processor. Examples are presented to illustrate how the damage measure estimate can be used to generate and update hypotheses of crack propagation based on the information of stress cycles. The effects of uncertainties in the initial conditions are included in the model in the construction of the probability distribution of damage measure.

Potential applications of the stochastic damage include the following technologies:

- Life extending control of mechanical systems $[25,39]$;

- Analytical measurements and intelligent sensing (including real-time non-destructive evaluation [17]) by on-line calibration of parameters of the stochastic damage measure model parameters of fatigue crack damage [14];

- Remaining life prediction of machinery components as well as generation of alerts and warnings for operational support and safety enhancement;

- Real-time maintenance decisions based on the information of machinery operation and anticipated usage.

A unified model that accounts for different sources (e.g., fracture geometry) of uncertainties in crack growth needs to be established before viable practical applications.

\section{Nomenclature}

$$
\begin{aligned}
a & =\text { crack length } \\
\mathrm{C} & =\text { autocovariance; covariance matrix } \\
\mathrm{c} & =\text { normalized crack length } \\
F(\cdot) & =\text { probability distribution function } \\
F & =\text { geometry factor for crack growth equation } \\
f(\cdot) & =\text { probability density function }
\end{aligned}
$$




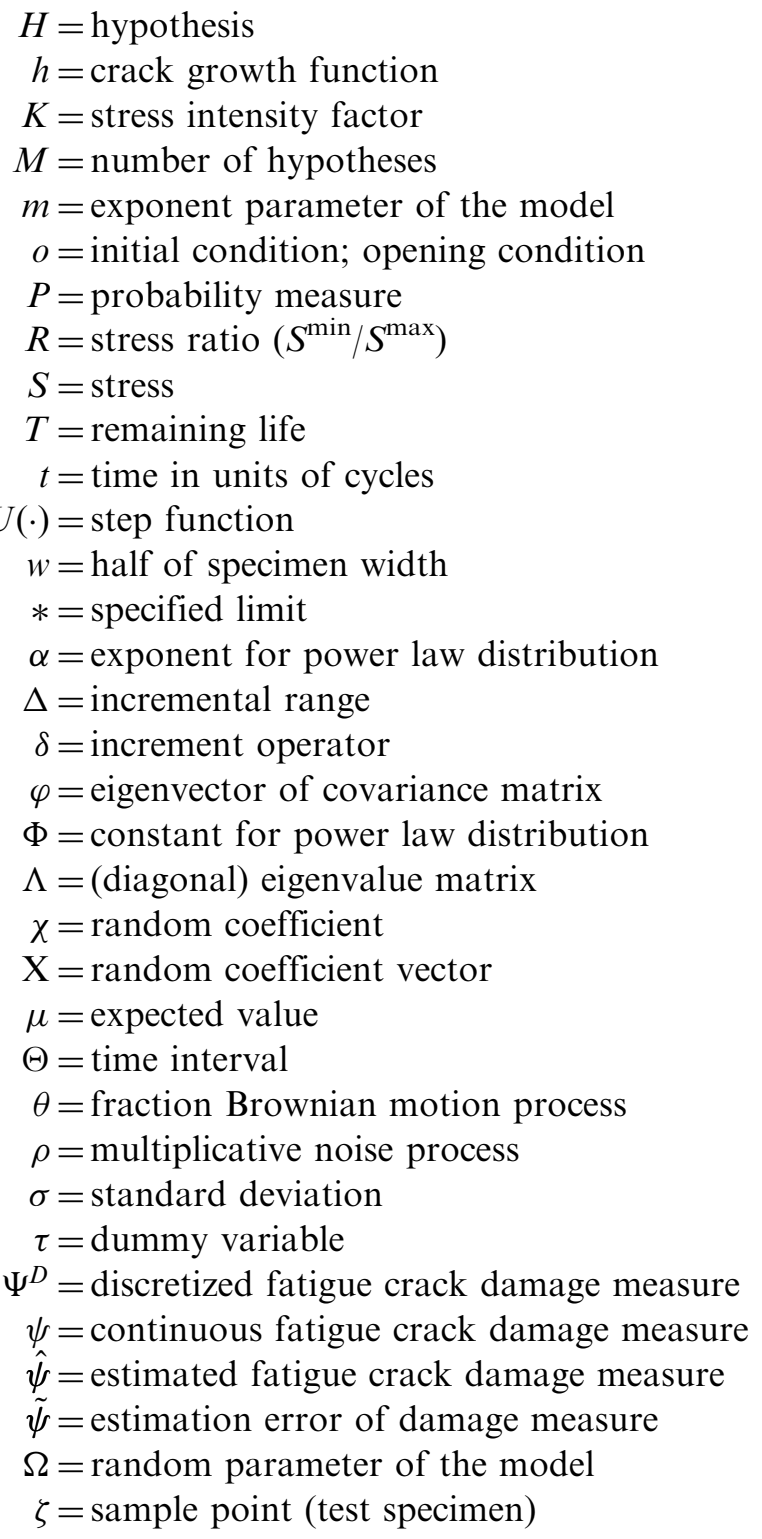

\section{Acknowledgments}

The author is grateful to Professor B.M. Hillberry of Purdue University and Professor H. Ghonem of University of Rhode Island for providing the test data of random fatigue crack growth. The work reported in this paper has been supported in part by the Army Research Office under Grant No. DAAD19-01-1-0646.

\section{Appendix: A Supporting Lemma}

Lemma: Let $A(\zeta)$ and $B(\zeta)$ be second-order real random variables; $\tilde{x}_{t}(\zeta)$ and $\tilde{y}_{t}(\zeta)$ be zeromean mean-square continuous (possibly nonseparable) real random processes; and the real $g(t)$ be almost everywhere continuous on an interval $\Theta$ such that, for all $t \in \Theta$, the following conditions hold:

(i) $A(\zeta) \stackrel{m s}{=} B(\zeta)$;

(ii) $E\left[A(\zeta) \tilde{x}_{t}(\zeta)\right]=0$ and $E\left[B(\zeta) \tilde{y}_{t}(\zeta)\right]=0$.

Then, the following mean-square identity

$$
A(\zeta) g(t)+\tilde{x}_{t}(\zeta) \stackrel{m s}{=} B(\zeta) g(t)+\tilde{y}_{t}(\zeta)
$$

yields

$$
\left.\begin{array}{l}
\tilde{x}_{t}(\zeta) \stackrel{m s}{=} \tilde{y}_{t}(\zeta) ; \\
E\left[A(\zeta) \tilde{y}_{t}(\zeta)\right]=0 ; \\
E\left[B(\zeta) \tilde{x}_{t}(\zeta)\right]=0
\end{array}\right\} \quad \forall t \in \Theta
$$

\section{Proof:}

It follows from the mean-square identity

$$
A(\zeta) g(t)+\tilde{x}_{t}(\zeta) \stackrel{m s}{=} B(\zeta) g(t)+\tilde{y}_{t}(\zeta)
$$

that

$$
\operatorname{Var}\left[(A(\zeta)-B(\zeta)) g(t)+\left(\tilde{x}_{t}(\zeta)-\tilde{y}_{t}(\zeta)\right)\right]=0
$$

that leads to:

$$
\begin{aligned}
& \operatorname{Var}[A(\zeta)-B(\zeta)] g^{2}(t)+\operatorname{Var}\left[\tilde{x}_{t}(\zeta)-\tilde{y}_{t}(\zeta)\right] \\
& \quad+E\left[(A(\zeta)-B(\zeta))\left(\tilde{x}_{t}(\zeta)-\tilde{y}_{t}(\zeta)\right)\right] g(t)=0
\end{aligned}
$$

A combination of Condition (i) and Schwarz inequality yields:

$$
\operatorname{Var}\left[\tilde{x}_{t}(\zeta)-\tilde{y}_{t}(\zeta)\right]=0
$$

and the remaining two identities follow from Condition (ii). 


\section{References}

1. Anderson, T.L. (1995). Fracture mechanics, 2nd Edn., CRC Press, Boca Raton, Florida.

2. Bartle, R. (1966). The elements of integration and lebesgue measure, John Wiley, New York.

3. Brunk, H.D. (1975). An introduction to mathematical statistics, 3rd Edn., Xerox Publishing, Lexington, MA.

4. Bolotin, V.V. (1989). Prediction of Service Life for Machines and Structures, ASME Press, New York.

5. Bogdanoff, J.L. and Kozin, F. (1985). Probabilistic models of cumulative damage, John Wiley, New York.

6. Casciati, F., Colombi, P., and Farvelli, L. (1992). Fatigue crack size probability distribution via a filter technique. Fatigue \& fracture of engineering materials \& structures, 15(5), 463-475.

7. Ditlevsen, O. (1986). Random fatigue crack growth - a first passage problem. Engineering Fracture Mechanics, 23(2), 467-477.

8. Fukunaga, K. (1990). Introduction to statistical pattern recognition, 2nd Edn., Academic Press, Boston.

9. Ghonem, H. and Dore, S. (1987). Experimental study of the constant probability crack growth curves under constant amplitude loading. Engineering Fracture Mechanics, 27, 1-25.

10. Holmes, M. and Ray, A. (1998). Fuzzy damage mitigating control of mechanical structures. ASME Journal of Dynamic Systems, Measurement and Control, 120(2), 249-256.

11. Hoyland, A. and Rausland, M. (1994). System reliability theory: models and statistical methods, WileyInterscience, New York.

12. Ishikawa, H., Tsurui, A., Tanaka, H. and Ishikawa, H. (1993). Reliability assessment based upon probabilistic fracture mechanics. Probabilistic Engineering Mechanics, 8, 43-56.

13. Jazwinski, A.H. (1970). Stochastic processes and filtering theory, Academic Press, New York.

14. Keller, E. and Ray, A. (2003). Real-time nondestructive evaluation of mechanical structures. Structural Health Monitoring, 2(3), 191-203.

15. Kloeden, P.E. and Platen, E. (1995). Numerical solution of stochastic differential equations, Springer, Berlin.

16. Lin, Y.K. and Yang, J.N. (1985). A stochastic theory of fatigue crack propagation. AIAA Journal, 23(1), 117-124.

17. Maldague, X.P.V. Ed. (1994). Advances in signal processing for nondestructive evaluation of materials, NATO ASI Series, Kluwer Academic Publications, Dordrecht, The Netherlands.
18. Mandelbrot, B. and Van Ness, J.W. (1968). Fractional brownian motion, fractional noises, and applications. SIAM Review, 10, 422-437.

19. Naylor, A.W. and Sell, G.R. (1982). Linear operator theory in engineering and science, Springer, New York.

20. Newman, J.C., Jr. (1984). A crack opening stress equation for fatigue crack growth. International Journal of Fracture, 24, R131-R135.

21. Newman, J.C., Jr. (1992). FASTRAN-II - A Fatigue Crack Growth Structural Analysis Program, NASA Technical Memorandum, Langley Research Center, Hampton, VA.

22. Oksendal, B. (2003). Stochastic Differential Equations: An Introduction with Applications, 6th Edn. Springer, Berlin.

23. Ozekici, S. ed. (1996). Reliability and Maintenance of Complex Systems, NATO Advanced Science Institutes (ASI) Series F: Computer and Systems Sciences, 154, Berlin, Germany.

24. Paris, P.C. and Erdogan, F. (1963). A critical analysis of crack propagation laws. Journal of Basic Engineering, Trans. ASME, D85, 528-534.

25. Ray, A. and Caplin, J. (2000). Life extending control of aircraft: Trade-off between flight performance and structural durability. The Aeronautical Journal, 104(1039), 397-408.

26. Ray, A. and Patankar, R. (1999). Stochastic modeling fatigue crack propagation under variable amplitude loading. Engineering Fracture Mechanics, 62, 477-493.

27. Ray, A. and Patankar, R. (2001). Fatigue crack growth under variable amplitude loading: Parts I and II. Applied Mathematical Modelling, 25, 979-1013.

28. Ray, A. and Phoha, S. (1999). Stochastic modeling of fatigue crack damage for information-based maintenance. Annals of Operation Research, 91, 197-204.

29. Ray, A. and Tangirala, S. (1997). A nonlinear stochastic model of fatigue crack dynamics. Probabilistic Engineering Mechanics, 12(1), 33-40.

30. Schijve, J. (1976). Observations on the Prediction of Fatigue Crack Growth Propagation Under VariableAmplitude Loading. Fatigue Crack Growth Under Spectrum Loads, ASTM STP, 595, 3-23.

31. Sobczyk, K. and Spencer, B.F. (1992). Random Fatigue: Data to Theory, Academic Press, Boston, MA.

32. Spencer, B.F., Tang, J. and Artley M.E. (1989). A stochastic approach to modeling fatigue crack growth. The AIAA Journal, 27(11), 1628-1635.

33. Suresh, S. (1991). Fatigue of materials, Cambridge University Press, Cambridge, UK.

34. Tsurui, A. and Ishikawa, H. (1986). Application of the Fokker-Planck to a stochastic fatigue growth model. Structural Safety, 4, 15-29. 
35. Virkler, D.A., Hillberry, B.M. and Goel, P.K. (1979). The statistical nature of fatigue crack propagation. ASME Journal of Engineering Materials and Technology, 101(2), 148-153.

36. West, B. (1999). Physiology, promisquity and prophecy at the millenium: A tale of tails, World Scientific, Singapore.

37. Wong, E. and Hajek, B. (1985). Stochastic processes in engineering systems, Springer-Verlag, New York.
38. Yang, J.N. and Manning, S.D. (1996). A simple second order approximation of stochastic crack growth analysis. Engineering Fracture Mechanics, 53(5), 677-686.

39. Zhang, H., Ray, A. and Phoha, S. (2000). Hybrid life extending control of mechanical systems: experimental validation of the concept. Automatica, 36(1), 23-36. 